\section{MDS: Weniger Bluttransfusionen erforderlich?}

\author{
Anämie ist bei myelodysplastischem Syndrom (MDS) mit niedrigem Risiko ein \\ häufiges Problem. In einer Studie wurde getestet, ob sich bei Patienten ohne \\ Deletion von Chromosom 5q (non-del[5q]) durch Lenalidomid die Häufigkeit \\ von Transfusionen reduzieren lässt.
}

\begin{abstract}
E rythropoese-stimulierende Agenzien - (ESA) bleiben bei Patienten mit Niedrigrisiko-MDS und non-del(5q) die Erstlinientherapie, jedoch nur bei einem Serum-Erythropoetin von $\leq 500 \mathrm{mU} / \mathrm{mL}$. Zudem geht das Ansprechen nach wiederholten Behandlungen häufig verloren. Deshabl wurde nun Lenalidomid bei diesen Patienten getestet. An der PhaseIII-Studie nahmen 239 transfusionsabhängige Patienten mit niedrig- oder intermediär-1-Risiko- und non-del(5q)MDS teil, die gegenüber ESA refraktär waren oder nicht dafür infrage kamen. Sie wurden 2:1 auf Lenalidomid oder Placebo 1-mal täglich randomisiert. Primärer Endpunkt war der Anteil transfusionsunabhängiger (RBC-TI) Patienten
\end{abstract}

enten aus der Lenalidomid-Gruppe hielt das Ansprechen zum Zeitpunkt der Analyse noch an (durchschnittlich 553 Tage). Bei niedrigeren Ausgangstitern an endogenem Erythropoetin ( $\leq 500 \mathrm{mU} /$ $\mathrm{mL}$ ) war die Ansprechrate doppelt so hoch wie bei höheren (34,0 vs. $15,5 \%)$.

In der 12. Woche unterschieden sich die Veränderungen der HRQoL zwischen den Therapiearmen nicht signifikant. Jedoch war das Erreichen von RBC-TI nach $\geq 8$ Wochen mit signifikanten HRQoLVerbesserungen verbunden $(\mathrm{p}<0,01)$.

Fazit: Durch die Anwendung von Lenalidomid konnte bei mehr als einem Viertel der transfusionsabhängigen Patienten mit non-del(5q)-MDS und niedrigerem Risiko anhaltende RBC-TI erreicht werden. Dies kann die HRQoL der Betroffenen verbessern. Kathrin von Kieseritzky

Santini V et al. Randomized Phase III Study of Lenalidomide Versus Placebo in RBC Transfusion-Dependent Patients With Lower-Risk Nondel(5q)Myelodysplastic Syndromes and Ineligible for or Refractory to Erythropoiesis-Stimulating Agents. J Clin Oncol. 2016;34(25):2988-96.

\title{
Erhaltungstherapie bei indolenten Lymphomen
}

\section{Bei Patienten mit indolenten Lymphomen hatte sich in der E1496-Studie die Erhaltungstherapie mit Rituximab nach Induktionschemotherapie als vorteilhaft herausgestellt. Nun wurden die Langzeitergebnisse präsentiert.}

$\mathrm{N}$ ach median 3,7 Jahren waren die ersten Ergebnisse der randomisierten Phase-III-Studie ECOG-ACRIN E1496 publiziert worden. Patienten, die nach der Induktionschemotherapie mit Cyclophosphamid, Vincristin und Prednison (CVP) auf eine Erhaltungstherapie mit Rituximab randomisiert worden waren, hatten im Vergleich zu nur beobachteten Patienten ein $60 \%$ geringeres Risikos für Progression oder Tod. Zudem wurde bei ihnen ein Trend zu einem verbesserten Gesamtüberleben (OS) beobachtet. Nun wurden die Ergebnisse nach median 11,5 Jahren veröffentlicht.

An der Studie nahmen 387 Patienten mit indolenten Lymphomen im Stadium III oder IV (nach Ann Arbor) teil. Sie erhielten zur Induktion CVP bis zum ma- ximalen Ansprechen, jedoch mindestens 6 und höchstens 8 Zyklen. Patienten mit mindestens einer Krankheitsstabilisierung wurden anschließend auf wöchentlich Rituximab (4-mal $375 \mathrm{mg} / \mathrm{m}^{2}$ alle 6 Monate über 2 Jahre; $\mathrm{n}=158$ ) oder Beobachtung $(\mathrm{n}=153)$ randomisiert, beginnend 4 Wochen nach Ende der Induktion. Primärer Endpunkt war das progressionsfreie Überleben (PFS).

Nach median 11,5 Jahren hatten die Patienten im Erhaltungsarm ein längeres medianes PFS (4,8 Jahre) als die Patienten der Beobachtungsgruppe (1,3 Jahre; Hazard Ratio [HR] 0,49; p < 0,0001). Im OS spiegelte sich das nicht wider (10-Jahres-OS-Rate 67 vs. $59 \%$; medianes OS 13,5 Jahre vs. Median nicht erreicht; HR 0,$91 ; p=0,69$ ). In der multivariaten Ana- lyse war die Erhaltungstherapie nicht signifikant mit dem PFS assoziiert, wohl aber die minimale Resterkrankung (MRD) nach Induktion (HR 0,71; p = $0,02)$. Eine niedrige initiale Tumorlast, MRD, follikuläre Histologie, ein niedriger FLIPI-Score und weibliches Geschlecht waren mit einem längeren $O S$ assoziiert. Es gab infolge der RituximabErhaltungstherapie im Vergleich zur Beobachtungsgruppe keine Steigerung der Rate an Zweitmalignomen.

Fazit: Im Langzeitverlauf beeinflusst die Erhaltungstherapie mit Rituximab das OS beim indolenten Lymphom nicht. Die Vorteile im PFS bleiben hingegen bestehen. Die Forscher empfehlen deshalb, die Erhaltungstherapie für Patienten mit indolentem B-Zell-Lymphom optional anzubieten.

Kathrin von Kieseritzky

Barta SK et al. Randomized phase 3 study in low-grade lymphoma comparing maintenance anti-CD20 antibody with observation after induction therapy: A trial of the ECOG-ACRIN Cancer Research Group (E1496). Cancer. 2016; 122(19):2996-3004. 